\title{
Secular instability in the spatial three-body problem
}

\author{
J. Féjoz, ${ }^{*}$ M. Guardia ${ }^{\dagger}$
}

May 21, 2015

\begin{abstract}
Consider the spatial three-body problem, in the regime where one body revolves far away around the other two, in space, the masses of the bodies being arbitrary but fixed; in this regime, there are no resonances in mean motions. The so-called secular dynamics governs the slow evolution of the Keplerian ellipses. We show that it contains a horseshoe and all the chaotic dynamics which goes along with it, corresponding to motions along which the eccentricity of the inner ellipse undergoes large, random excursions. The proof goes through the surprisingly explicit computation of the homoclinic solution of the first order secular system, its complex singularities and the Melnikov potential.
\end{abstract}

\section{Introduction}

The question of the stability of the Solar System is the oldest open problem in Dynamical Systems. A number of works have shown striking instability mechanisms in the threebody problems, e.g. oscillatory orbits and close to parabolic motion Ale69, Sit60, LS80a, LS80b, GMS13, DKdIRS14, chaotic dynamics near double or triple collisions Bol06, Moe89] (see also Mos01]). But scarce mathematical mechanisms have been described regarding more astronomical regimes, which would be plausible for subsystems of solar or extra-solar systems. One of them [FGKR15] shows the existence of global instabilities along mean motion (i.e. Keplerian) resonances.

In this paper, we focus on secular resonances. Numerical evidence has long been suggesting that such resonances are a major source of chaos in the Solar system [LR93, Las08, FS89. For example, astronomers have established that Mercury's eccentricity is chaotic and can increase so much that collisions with Venus or the Sun become possible, as a result from an intricate network of secular resonances [BLF12]. On the other hand, that Uranus's obliquity $\left(97^{\circ}\right)$ is essentially stable, is explained, to a large extent, by the absence of any low-order secular resonance [BL10, LR93. It is the goal of this paper to provide a simple instability mechanism in the secular dynamics of the three-body problem.

\footnotetext{
${ }^{*}$ Université Paris-Dauphine (Ceremade) \& Observatoire de Paris (IMCCE), France, jacques.fejoz@dauphine.fr

${ }^{\dagger}$ Universitat Politècnica de Catalunya (Departament de Matemàtica Aplicada I), Spain, marcel.guardia@upc.edu.
} 


\section{The secular Hamiltonian in the lunar regime}

Consider three point masses undergoing the Newtonian attraction in space. Call $m_{0}, m_{1}$ and $m_{2}$ the masses and $\left(q_{j}, p_{j}\right)_{j=1,2,3} \in\left(\mathbb{R}^{3} \times \mathbb{R}^{3}\right)^{3}$ the Jacobi coordinates. After having symplectically reduced the system by the symmetry of translations, the Hamiltonian depends only on $\left(q_{j}, p_{j}\right)_{j=1,2}$ and equals

$$
F_{\text {Kep }}+F_{\mathrm{per}}, \quad\left\{\begin{array}{l}
F_{\mathrm{Kep}}=\sum_{j=1,2}\left(\frac{p_{j}^{2}}{2 \mu_{j}}-\frac{\mu_{j} M_{j}}{\left\|q_{j}\right\|}\right) \\
F_{\mathrm{per}}=\left(\frac{\mu_{2} M_{2}}{\left\|q_{2}\right\|}-\frac{m_{0} m_{2}}{\left\|q_{2}+\sigma_{1} q_{1}\right\|}-\frac{m_{1} m_{2}}{\left\|q_{2}-\sigma_{0} q_{1}\right\|}\right)
\end{array}\right.
$$

where the reduced masses are defined by

$$
M_{0}=m_{0}, \quad M_{1}=m_{0}+m_{1}, \quad \mu_{1}^{-1}=M_{0}^{-1}+m_{1}^{-1}, \quad \mu_{2}^{-1}=M_{1}^{-1}+m_{2}^{-1}
$$

and coefficients $\sigma_{j}$ are the barycentric weights of the inner bodies: $\sigma_{j}=m_{j} / M_{1}(j=$ $0,1) . F_{\text {Kep }}$ is the integrable Hamiltonian of two uncoupled Kepler problems and induces a "Keplerian" action of the 2-torus, while $F_{\text {per }}$ is the so-called perturbing function.

In this paper, we consider the asymptotic regime (sometimes called lunar or hierarchical or stellar) where the masses are fixed, while body 2 revolves far away around the other two. In particular, each of the two terms of $F_{\text {Kep }}$ is negative, the outer eccentricity is bounded away from 1 , and the semi major axes $a_{1}$ of $q_{1}$ and $a_{2}$ of $q_{2}$ satisfy

$$
a_{1}=O(1) \ll a_{2} \rightarrow \infty .
$$

The expansion of $F_{\text {per }}$ in the powers of $\left\|q_{1}\right\| /\left\|q_{2}\right\| \ll 1$ is

$$
F_{\mathrm{per}}=-\frac{\mu_{1} m_{2}}{\left\|q_{2}\right\|} \sum_{n \geq 2} \sigma_{n} P_{n}(\cos \zeta)\left(\frac{\left\|q_{1}\right\|}{\left\|q_{2}\right\|}\right)^{n},
$$

where the $P_{n}$ 's are the Legendre polynomials, and $\sigma_{n}=\sigma_{0}^{n-1}+(-1)^{n} \sigma_{1}^{n-1}, n \geq 2$. Note that the perturbing function is of the order of $1 / a_{2}^{3}$.

Standard normal form theory 1 shows that, for every integer $k$, there is a local change of coordinates $a_{2}^{-3}$-close to the identity which transforms the Hamiltonian into

$$
F=F_{\mathrm{Kep}}+F_{\mathrm{sec}}+O\left(a_{2}^{-k-1}\right),
$$

where $F_{\text {sec }}$ is the secular Hamiltonian 2 of order $k$

$$
F_{\mathrm{sec}}=\int_{\mathbb{T}^{2}} F_{\mathrm{per}} d \ell_{1} d \ell_{2}+O\left(\frac{1}{a_{2}}\right)
$$

obtained by averaging out the mean anomalies $\ell_{j}$ at successive orders; $\mathbb{T}^{2}$ is the orbit of the Keplerian action defined by $F_{\text {Kep }}$, parameterized by the mean anomalies $\ell_{1}$ and $\ell_{2}$ of the two planets. The secular Hamiltonian descends to the quotient by the Keplerian action of $\mathbb{T}^{2}$, and induces a Hamiltonian system on the space of pairs of Keplerian ellipses with fixed semi major axes, describing the slow evolution of the Keplerian ellipses due to the perturbation.

\footnotetext{
${ }^{1}$ See, in this context, Féj02 : mean motions do not interfere since they are not of the same order.

${ }^{2}$ Secular, means century in Latin. This term governs the slow dynamics (as opposed to the fast, Keperian dynamics), on a long time scale, symbolically one century.
} 
In this paper, we primarily consider the principal part of the Hamiltonian $F$ of equation (3), i.e. $F_{\text {Kep }}+F_{\text {sec }}$.

We establish the phase portrait of the secular system, in particular with a (well known) hyperbolic periodic orbit located at inclined and nearly circular ellipses. The main results of the paper are the following. We prove that this periodic orbit posesses transversal homoclinic points (Corollary 4.2) and that the secular system posesses a horseshoe (Theorem 4.3), and therefore chaotic motions. As a by-product, we prove that the secular system is non-integrable. The results we obtain are valid for any value of the masses of the three bodies.

To prove these results, we take advantage of the fact that the first order in $1 / a_{2}$ of the secular Hamiltonian is integrable. We explicitely determine the homoclinic solution to the hyperbolic periodic orbit of this first order (Lemma 3.1). Then, we show that the Melnikov potential associated with this homoclinic orbit has non-degenerate critical points (Proposition 4.1), from what it follows that the secular homoclinic solution splits for the full secular system, as well as for the full initial system.

The result we present in this paper is a step towards proving the existence of unstable orbits in the three body problem in the lunar regime, for any value of the masses of the bodies, in the sense of Arnold diffusion. By "unstable orbits" here we mean orbits such that one of the three bodies undergoes a large change in the semi major axis of the associated osculating ellipse. This is explained more precisely in Section 4.1.

\subsection{The quadrupolar and octupolar Hamiltonians}

The first two terms of the expansion (2) of $F_{\text {per }}$, after averaging, yield the quadrupolar and octupolar Hamiltonians: 3

$$
F_{\mathrm{sec}}=-\mu_{1} m_{2}\left(F_{\text {quad }}+\left(\sigma_{0}-\sigma_{1}\right) F_{\mathrm{oct}}\right)+O\left(\frac{a_{1}^{4}}{a_{2}^{5}}\right),
$$

with

$$
F_{\text {quad }}=\frac{a_{1}^{2}}{8 a_{2}^{3}\left(1-e_{2}^{2}\right)^{3 / 2}}\left(\left(15 e_{1}^{2} \cos ^{2} g_{1}-12 e_{1}^{2}-3\right) \sin ^{2} i+3 e_{1}^{2}+2\right)
$$

and

$$
\begin{gathered}
F_{\text {oct }}=-\frac{15}{64} \frac{a_{1}^{3}}{a_{2}^{4}} \frac{e_{1} e_{2}}{\left(1-e_{2}^{2}\right)^{5 / 2}} \times \\
\left\{\begin{array}{l}
\cos g_{1} \cos g_{2}\left[\begin{array}{l}
\frac{G_{1}^{2}}{L_{1}^{2}}\left(5 \sin ^{2} i\left(-7 \cos ^{2} g_{1}+6\right)-3\right) \\
-35 \sin ^{2} g_{1} \sin ^{2} i+7
\end{array}\right] \\
-\sin g_{1} \sin g_{2} \cos i\left[\begin{array}{l}
\frac{G_{1}^{2}}{L_{1}^{2}}\left(5 \sin ^{2} i\left(7 \cos ^{2} g_{1}-4\right)+3\right) \\
+35 \sin ^{2} g_{1} \sin ^{2} i-7
\end{array}\right]
\end{array}\right\} .
\end{gathered}
$$

this is a standard computation (see [Féj02] for the reduction to integrating trigonometric polynomials), and we have used the following notations:

$$
\begin{cases}e_{j} & =\text { eccentricities } \\ g_{j} & =\text { arguments of pericenters } \\ i & =\text { mutual inclination. }\end{cases}
$$

\footnotetext{
${ }^{3}$ More generally, the $n$-th order term is called $2^{n}$-polar, because, in electrostatic, this term is the dominating term of the potential of a system of $2^{n}$ well chosen charged particles.
} 
The Hamiltonians $F_{\text {quad }}$ and $F_{\text {oct }}$ do not depend on the order $k$ of averaging in the expression (3) (i.e., due to the special dependence of $F_{\text {Kep }}$ and $F_{\text {per }}$ in $L_{2}$, these first two terms of the normal form are not modified by second and higher order averaging).

Recall that we want to analyze the secular Hamiltonian $F_{\text {sec }}$ for any value of the masses, so that parameters $m_{i}, \mu_{i}$ and $M_{i}$ are just given constants satisfying (1).

Let $\left(\ell_{j}, L_{j}, g_{j}, G_{j}, \theta_{j}, \Theta_{j}\right)_{j=1,2}$ denote the Delaunay variables. Jacobi's classical elimination of the node consists in considering a codimension-3 submanifold of fixed, vertical angular momentum, and quotienting by horizontal rotations. The reduced manifold has dimension 8, on which the Keplerian and eccentric variables $\left(\ell_{j}, L_{j}, g_{j}, G_{j}\right)_{j=1,2}$ induce symplectic coordinates. After averaging out the mean motions we are left with the symplectic coordinates $\left(g_{j}, G_{j}\right)_{j=1,2}$, and the variables $L_{j}$ may be treated as parameters.

The variables $L_{i}$ are given by $L_{i}=\mu_{i} \sqrt{M_{i} a_{i}}$. Therefore, in the lunar regime we have $L_{1} \sim 1$ and $L_{2} \gg 1$. Recall that the eccentricity of the ellipse is given in terms of the Delaunay coordinates by

$$
e_{i}=\sqrt{1-\frac{G_{i}^{2}}{L_{i}^{2}}} .
$$

Since we want the outer body to describe non-degenerate ellipses, we even assume $G_{2} \sim$ $L_{2}$. Since we are doing a perturbative analysis in $L_{2}^{-1}$, we define the new variable

$$
\Gamma=C-G_{2} \text {, where } C=\delta L_{2} \text { and } \delta>0 \text { is a fixed constant. }
$$

The coordinates

$$
\left(g_{1}, G_{1}, \gamma, \Gamma\right)=\left(g_{1}, G_{1},-g_{2}, C-G_{2}\right),
$$

are symplectic; we also call them Delaunay coordinates (as opposed to some radically different coordinates used later). Now these variables are bounded as $a_{2}, L_{2} \rightarrow \infty$ (recall that $\left.C \sim G_{2} \rightarrow \infty\right)$. At the first order in $L_{2}^{-1}$, the mutual inclination $i$ satisfies

$$
\cos ^{2} i=\frac{\left(C^{2}-G_{1}^{2}-G_{2}^{2}\right)^{2}}{4 G_{1}^{2} G_{2}^{2}}=\frac{\left(C^{2}-G_{1}^{2}-(C-\Gamma)^{2}\right)^{2}}{4 G_{1}^{2}(C-\Gamma)^{2}}=\frac{\Gamma^{2}}{G_{1}^{2}}+O\left(L_{2}^{-1}\right) .
$$

Now we express the secular Hamiltonian in these new variables and expand it in inverse powers of $L_{2}$. Recall that variables $L_{j}$ are parameters, as well as the norm $C$ of the angular momentum.

Lemma 2.1. The secular Hamiltonian (5) has the form

$$
F_{\mathrm{sec}}=\alpha_{0}+\alpha_{1} L_{2}^{-6}\left(H_{0}+L_{2}^{-1} H_{1}+\left(\sigma_{0}-\sigma_{1}\right) L_{2}^{-2} H_{2}+\mathcal{O}\left(L_{2}^{-3}\right)\right) .
$$

with

$$
H_{0}=\left(1-\frac{G_{1}^{2}}{L_{1}^{2}}\right)\left[2-5\left(1-\frac{\Gamma^{2}}{G_{1}^{2}}\right) \sin ^{2} g_{1}\right]-\frac{\Gamma^{2}}{L_{1}^{2}}
$$

In the coordinates $\left(g_{1}, G_{1}, \gamma, \Gamma\right), F_{\text {quad }}$ and hence $H_{0}$ and $H_{1}$ do not depend on $\gamma$. We do not compute $H_{1}$ explicitly, here. The reason is that $H_{1}$ does not break the integrability of $H_{0}$ and therefore does not play any role in the Melnikov analysis. In contrast, the Hamiltonian $\mathrm{H}_{2}$ does break integrability (as it will follow from our study), and is computed in Section 5 .

Proof of Lemma 2.1. Formula (8) implies

$$
\frac{1}{G_{1}^{2}}=\frac{e_{1}^{2}}{G_{1}^{2}}+\frac{1}{L_{1}^{2}} .
$$


Hence, using (10),

$$
\sin ^{2} i=1-\frac{\Gamma^{2}}{G_{1}^{2}}+O\left(L_{2}^{-1}\right)=1-\Gamma^{2} \frac{e_{1}^{2}}{G_{1}^{2}}-\frac{\Gamma^{2}}{L_{1}^{2}}+O\left(L_{2}^{-1}\right) .
$$

Besides,

$$
e_{2}=\sqrt{1-\frac{G_{2}^{2}}{L_{2}^{2}}}=\sqrt{1-\frac{C^{2}}{L_{1}^{2}}}+O\left(L_{2}^{-1}\right)
$$

is a first integral of the first order approximation of $F_{\text {quad }}$. Hence, expanding in powers of $L_{2}^{-1}, F_{\text {quad }}$ can be written as

$$
F_{\text {quad }}\left(g_{1}, G_{1}, \Gamma\right)=\alpha_{1} L_{2}^{-6}\left(H_{0}\left(g_{1}, G_{1}, \Gamma\right)+2\right)+O\left(L_{2}^{-1}\right)
$$

with

$$
\alpha_{1}=-\frac{L_{1}^{4} M_{2} \mu^{6} m_{2}}{8 M_{1}^{2} \mu_{1}^{3}\left(1-e_{2}^{2}\right)^{3 / 2}}
$$

and

$$
\begin{aligned}
H_{0} & =\left(5 e_{1}^{2} \cos ^{2} g_{1}-4 e_{1}^{2}-1\right) \sin ^{2} i+\left.e_{1}^{2}\right|_{L_{2}^{-1}=0} \quad(\text { using (6) }) \\
& =e_{1}^{2}\left[\left(5 \cos ^{2} g_{1}-4\right)\left(1-\frac{\Gamma^{2}}{G_{1}^{2}}\right)+\frac{\Gamma^{2}}{G_{1}^{2}}+1\right]-\frac{\Gamma^{2}}{L_{1}^{2}} \quad(\text { using (10) } \text { and (13) }) \\
& =e_{1}^{2}\left[2-5\left(1-\frac{\Gamma^{2}}{G_{1}^{2}}\right) \sin ^{2} g_{1}\right]-\frac{\Gamma^{2}}{L_{1}^{2}} .
\end{aligned}
$$

Factorization (12) follows.

\section{The quadrupolar phase portrait}

According to (6), the quadrupolar Hamiltonian $F_{\text {quad }}$ and thus $H_{0}$ do not to depend on $\gamma$. This happy coincidence (which does not repeat itself for the next order term $F_{\text {oct }}$ ) makes $F_{\text {quad }}$ integrable. This has been extensively used (see [Zha14] and references therein). Here, we may thus think of $\Gamma$ as a parameter.

Complex singularities of solutions of $F_{\text {quad }}$ are hard to determine in general. In our regime, the first order of the quadrupolar Hamiltonian, $H_{0}$, can be factorized as described in equation (12) (up to the additive constant $-\Gamma^{2} / L_{1}^{2}$ ), which dramatically simplifies our study.

The Hamiltonian $H_{0}$ is analytic on a neighborhood of the cylinder

$$
\left.\left(g_{1}, G_{1}\right) \in \mathbb{T}^{1} \times\right] 0, L_{1}[
$$

in $\mathbb{T}^{1} \times \mathbb{R}$. Since it is $\pi$-periodic with respect to $g_{1}$, we may focus on the domain $0 \leq g_{1} \leq \pi, 0 \leq G_{1} \leq L_{1}$, keeping in mind that the Delaunay coordinates blow up circular ellipses $\left(G_{1}=L_{1}\right)$.

Hamilton's vector field is

$$
\left\{\begin{array}{l}
\dot{g}_{1}=\frac{2 G_{1}}{L_{1}^{2}}\left[5\left(1-\frac{\Gamma^{2}}{G_{1}^{2}}\right) \sin ^{2} g_{1}-2\right]-10\left(1-\frac{G_{1}^{2}}{L_{1}^{2}}\right) \frac{\Gamma^{2}}{G_{1}^{3}} \sin ^{2} g_{1} \\
\dot{G}_{1}=5\left(1-\frac{G_{1}^{2}}{L_{1}^{2}}\right)\left(1-\frac{\Gamma^{2}}{G_{1}^{2}}\right) \sin 2 g_{1} .
\end{array}\right.
$$

The second component $\dot{G}_{1}$ vanishes if and only if (assuming $G_{1}>0$ )

$$
G_{1} \in\left\{|\Gamma|, L_{1}\right\} \quad \text { or } \quad g_{1}=0 \quad(\bmod \pi / 2) .
$$


If $G_{1}=|\Gamma|, \dot{g}_{1}$ cannot vanish. Moreover, $\left.G_{1} \in\right] 0, L_{1}[$. Let

$$
\left.\tilde{\Gamma}=\sqrt{1-\frac{\Gamma^{2}}{L_{1}^{2}}} \in\right] 0,1[.
$$

The equilibrium points thus satisfy the following equations:

- If $G_{1}=L_{1}$ (circular ellipse),

$$
\sin ^{2} g_{1}=\frac{2}{5 \tilde{\Gamma}^{2}}
$$

Assuming that

$$
|\Gamma|<L_{1} \sqrt{\frac{3}{5}},
$$

whence $\tilde{\Gamma}^{2} \geq \frac{2}{5}$ (this condition is satisfied if the inner ellipse has a large eccentricity or an inclination close to $\pi / 2)$, within the $g_{1}$-interval $[0, \pi[$, there are two solutions $\left.g_{1}^{\min } \in\right] 0, \pi / 2\left[\right.$ and $g_{1}^{\max }$ symmetric with respect to $\pi / 2$, which are located on the energy level $H_{0}=-\Gamma^{2} / L_{1}^{2}$.

In variables $(x, y)$ such that

$$
\sin ^{2} g_{1}=\frac{2}{5 \tilde{\Gamma}^{2}}(1+x) \quad \text { and } \quad G_{1}=L_{1}(1-y)
$$

(which are local coordinates in the neighborhood of either one of the two above singularities), we have

$$
H_{0}+\Gamma^{2} / L_{1}^{2}=4 x y+O_{3}(x, y)
$$

thus the two singularities are hyperbolic.

- If $g_{1}=0(\bmod \pi), G_{1}=0$ (collision ellipse) is an equilibrium point of the regularized Hamiltonian vector field $G_{1}^{3} X_{F_{\text {quad }}^{0}}$.

- If $g_{1}=\pi / 2(\bmod \pi)$,

$$
3 G_{1}^{4}-10 \Gamma^{2} G_{1}^{2}+5 L_{1}^{2} \Gamma^{2}=0
$$

(two pairs of opposite real solutions).

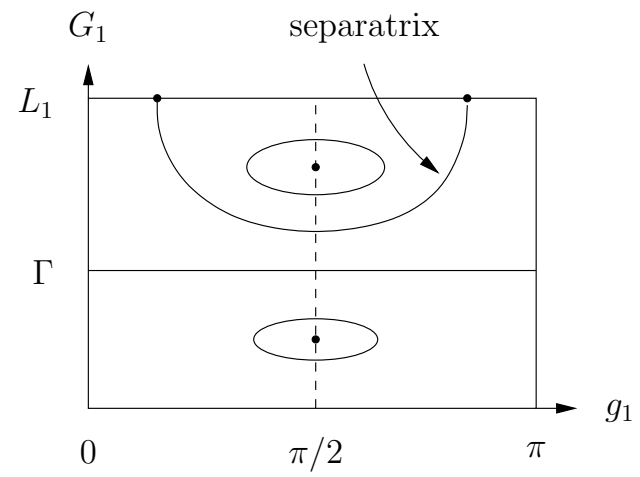

Figure 1: Phase portrait of the reduced quadrupolar dynamics 
We focus on the first two hyperbolic singularities, with $G_{1}=L_{1}$, henceforth assuming that condition (16) is satisfied, with, say,

$$
\Gamma>0 \text {. }
$$

Lifted to the full secular phase space, these critical points become the periodic orbits

$$
Z_{\min , \max }^{0}\left(t, \gamma_{0}\right)=\left(g_{1}^{\min , \max }, L_{1}, \gamma^{0}+\gamma^{1}(t), \Gamma\right),
$$

where $\gamma^{0}$ is just the initial condition for the variable $\gamma$ and

$$
\gamma^{1}(t)=-2 \frac{\Gamma}{L_{1}^{2}} t
$$

The whole circle $G_{1}=L_{1}$ corresponds to circular motion. Along this circle the Delaunay variables are singular. Thus, in a neighborhood of circular motion it is more reliable to use the Poincaré variables

$$
\left\{\begin{array}{l}
\xi=\sqrt{2\left(L_{1}-G_{1}\right)} \cos g_{1} \\
\eta=-\sqrt{2\left(L_{1}-G_{1}\right)} \sin g_{1}
\end{array}\right.
$$

Those secular coordinates are symplectic (a well known fact). The Poincaré variables transform the Hamiltonian $H_{0}$ into

$$
\widetilde{H}_{0}(\xi, \eta)=-\frac{\Gamma^{2}}{L_{1}^{2}}+\frac{1}{L_{1}}\left[2 \xi^{2}-\left(3-5 \frac{\Gamma^{2}}{L_{1}^{2}}\right) \eta^{2}\right]+\mathcal{O}_{2}\left(\xi^{2}+\eta^{2}\right)
$$

and the line segment $\left\{G_{1}=L_{1}\right\}$ blows down to a single hyperbolic periodic orbit

$$
(\xi, \eta, \gamma, \Gamma)=\left(0,0, \gamma^{0}+\gamma^{1}(t), \Gamma\right)
$$

in the secular space.

As it has occured above, in the $\left(g_{1}, G_{1}\right)$-plane there are two hyperbolic fixed points for $g_{1} \in[0, \pi]$ and two more for $g_{1} \in[\pi, 2 \pi]$. On the line $G_{1}=L_{1}$, there are heteroclinic connections between them. All these objects blow down to the critical point $(\xi, \eta)=$ $(0,0)$ in Poincaré variables. Moreover, there are two separatrix connections in the region $G_{1}<L_{1}$, one between the critical points with $g_{1} \in[0, \pi]$ and the other one between the critical points with $g_{1} \in[\pi, 2 \pi]$. Even if $H_{0}$ is well defined for $G_{1}>L_{1}$, the corresponding solutions are spurious. In the secular space, we obtain a hyperbolic critical point at $(\xi, \eta)=(0,0)$ with two homoclinic orbits forming a figure eight.

The main technical goal of this paper is to show that these separatrices split when one considers the complete secular Hamiltonian of arbitrary order $k$. We focus on the homoclinic on which $g_{1} \in[0, \pi]$. We use both Delaunay and Poincaré variables, but need to keep in mind that the Delaunay variables are not defined on the secular space proper along circular inner ellipses $\left(G_{1}=L_{1}\right)$. Note the same orbit is homoclinic for the secular Hamiltonian (21) and heteroclinic after blow up (see (12)).

The expression of the energy (12) yields the following characterization of this orbit, where the interval $] g_{1}^{\min }, g_{1}^{\max }[\subset] 0, \pi[$ is defined by the inequality (recall (14))

$$
\sin ^{2} g_{1}>\frac{2}{5 \tilde{\Gamma}^{2}}
$$

and

$$
\chi=\sqrt{\frac{2}{3}} \frac{|\Gamma|}{L_{1}} \frac{1}{\sqrt{1-\frac{5}{3} \frac{\Gamma^{2}}{L_{1}^{2}}}}>0 \quad \text { and } \quad A_{2}=30 \sqrt{\frac{10}{3}} \frac{|\Gamma|^{3}}{L_{1}} \sqrt{1-\frac{5}{3} \frac{\Gamma^{2}}{L_{1}^{2}}}
$$


Lemma 3.1. Assume conditions (16)-(17). The Hamiltonian $H_{0}$ given in (12) has a heteroclinic solution which tends to the periodic orbits $Z_{\min }^{0}$ and $Z_{\max }^{0}$ in (18) in backward and forward times respectively. Its orbit is defined by the equation

$$
\left(1-\frac{\Gamma^{2}}{G_{1}^{2}}\right) \sin ^{2} g_{1}=\frac{2}{5}
$$

with range $\left.g_{1} \in\right] g_{1}^{\min }, g_{1}^{\max }[\subset] 0, \pi[$. Its time parameterization is given by

$$
Z^{0}\left(t, \gamma^{0}\right)=\left(g_{1}^{h}(t), G_{1}^{h}(t), \gamma^{h}(t), \Gamma\right)
$$

with

$$
\begin{aligned}
\cos g_{1}^{h}(t) & =-\sqrt{\frac{3}{5}} \frac{\sinh A_{2} t}{\sqrt{\chi^{2}+\left(1+\chi^{2}\right) \sinh ^{2} A_{2} t}} \\
G_{1}^{h}(t) & =|\Gamma| \sqrt{\frac{5}{3}} \sqrt{1+\frac{3}{5} \frac{L_{1}^{2}}{\Gamma^{2}} \sinh ^{2} A_{2} t}\left(\cosh A_{2} t\right)^{-1} \\
\gamma^{h}(t) & =\gamma^{0}+\gamma^{1}(t)+\gamma^{2}(t), \quad \gamma^{2}(t)=\arctan \left(\chi^{-1} \tanh A_{2} t\right),
\end{aligned}
$$

where $\gamma^{0} \in \mathbb{T}$ is the arbitrary value of the $\gamma$ coordinate at initial time, $\gamma^{1}$ has been introduced in (19).

In the $\gamma$-component of the separatrix, the angle $-\gamma^{0}$ is the (arbitrary) argument of the outer pericenter at the symmetry center of the separatrix $g_{1}=\pi / 2$ (recall the change of coordinates (9) ). The term $\gamma^{1}(t)$ is the rotational part and the term $\gamma^{2}(t)$ is the transient part of $\gamma^{h}(t)$.

Remark 3.2. The assumed condition (16) implies that $\partial_{\Gamma} H_{0} \neq 0$ in a neighborhood of the heteroclinic orbit. Therefore, one can rephrase Lemma 3.1 as the existence of a heteroclinic orbit in each energy level $H_{0}\left(g_{1}, G_{1}, \gamma, \Gamma\right)=h$ for any $h<0$ (recall that by the expression of $H_{0}$ in (12) the heteroclinic orbits always have negative energy).

Proof. On the separatrix, we have

$$
\dot{g}_{1}=10 \Gamma^{2} \frac{1-G_{1}^{2} / L_{1}^{2}}{G_{1}^{3}} \sin g_{1}^{2} .
$$

Using (24) and (22), one can eliminate $G_{1}$ by

$$
G_{1}=\frac{|\Gamma| \sqrt{5} \sin g_{1}}{\sqrt{3-5 \cos ^{2} g_{1}}}
$$

and get a closed differential equation

$$
\dot{g}_{1}=A_{1} \frac{\left(1-\frac{5}{3}\left(1+\chi^{2}\right) \cos ^{2} g_{1}\right) \sqrt{1-\frac{5}{3} \cos ^{2} g_{1}}}{\sin g_{1}}
$$

where $\chi$ has been defined in (23) and

$$
A_{1}=30 \sqrt{3} \Gamma^{2}\left(1-\frac{5}{3} \frac{\Gamma^{2}}{L_{1}^{2}}\right)>0 .
$$


Note that, due to $\Gamma \neq 0$ and (22),$\dot{g}_{1} \neq 0$ along the separatrix. So, one can separate variables and, choosing $g_{1}=\pi / 2$ at $t=0$ yields

$$
A_{1} t=\int_{\pi / 2}^{g_{1}} \frac{\sin g_{1} d g_{1}}{\left(1-\frac{5}{3}\left(1+\chi^{2}\right) \cos ^{2} g_{1}\right) \sqrt{1-\frac{5}{3} \cos ^{2} g_{1}}} .
$$

The variable $\left.\cos g_{1} \in\right]-\frac{\sqrt{3}}{\sqrt{5\left(1+\chi^{2}\right)}}, \frac{\sqrt{3}}{\sqrt{5\left(1+\chi^{2}\right)}}[$ is a coordinate on the separatrix. Let $x$ be defined by

$$
\left.\sqrt{\frac{5}{3}} \cos g_{1}=\cos x \in\right] 0, \frac{1}{\left(1+\chi^{2}\right)}[\text {. }
$$

Then

$$
\begin{aligned}
A_{1} t & =\sqrt{\frac{3}{5}} \int_{\pi / 2}^{\arccos \sqrt{\frac{5}{3}} \cos g_{1}} \frac{d x}{1-\left(1+\chi^{2}\right) \cos ^{2} x} \\
& =\frac{1}{2 \chi} \sqrt{\frac{3}{5}} \ln \frac{\tan x-\chi}{\tan x+\chi}, \quad \text { with } \cos x=\sqrt{\frac{5}{3}} \cos g_{1} .
\end{aligned}
$$

One can check that the constant $A_{2}$ defined in (23) satisfies $A_{2}=\sqrt{\frac{5}{3}} A_{1} \chi$. The equation can be solved for $\tan x$, which yields $\tan x=-\chi \operatorname{coth}\left(A_{2} t\right)$. This equality, jointly with

$$
\cos g_{1}=\sqrt{\frac{3}{5}} \cos x=\sqrt{\frac{3}{5}} \frac{ \pm 1}{\sqrt{1+\tan ^{2} x}}
$$

gives formula (26). Using (29), the $G_{1}$-component of the separatrix given in (27) can be easily obtained. Finally, only the $\gamma$-component remains to be computed. But, along the separatrix solution, $\dot{\gamma}=\partial_{\Gamma} H_{0}$. Using (12), (26) and (29),

$$
\begin{aligned}
\dot{\gamma} & =10 \frac{\Gamma}{G_{1}^{2}}\left(1-\frac{G_{1}^{2}}{L_{1}^{2}}\right) \sin ^{2} g_{1}-2 \frac{\Gamma}{L_{1}^{2}} \\
& =\frac{4 \Gamma}{L_{1}^{2} \chi^{2}}\left(1-\frac{5}{3}\left(1+\chi^{2}\right) \cos ^{2} g_{1}\right)-2 \frac{\Gamma}{L_{1}^{2}} \\
& =\frac{4 \Gamma}{L_{1}^{2}} \frac{1}{\left(\left(1+\chi^{2}\right) \cosh ^{2} A_{2} t-1\right)}-2 \frac{\Gamma}{L_{1}^{2}} .
\end{aligned}
$$

Since $\frac{4 \Gamma}{L_{1}^{2} \chi A_{2}}=\frac{4 \Gamma \sqrt{3}}{L_{1}^{2} \chi^{2} A \sqrt{5}}=1$, equation (28) follows.

\section{Splitting of separatrices}

Lemma 3.1 shows that the Hamiltonian $H_{0}$ in (6) has a heteroclinic connection (homoclinic in the secular space for the Hamiltonian (21)).

The next term in the asymptotic expansion of the secular Hamiltonian given in Lemma 2.1 does not depend on $\gamma$ and therefore does not break integrability. For the amended Hamiltonian, according to classical perturbation theory, the hyperbolic periodic orbit and the homoclinic orbit persist. Moreover, the periodic orbit remains at $(\xi, \eta)=$ $(0,0)$. Thus, in Delaunay coordinates, there are two hyperbolic periodic orbits, which are of the form

$$
\left\{\begin{array}{l}
Z_{\min }^{\varepsilon}\left(t, \gamma^{0}\right)=\left(g_{\min }+O(\varepsilon), L_{1}, \gamma^{0}+\gamma^{1}(t)+O(\varepsilon), \Gamma\right) \\
Z_{\max }^{\varepsilon}\left(t, \gamma^{0}\right)=\left(g_{\max }+O(\varepsilon), L_{1}, \gamma^{0}+\gamma^{1}(t)+O(\varepsilon), \Gamma\right)
\end{array}\right.
$$


where $\varepsilon=L_{2}^{-1}$. We can choose the time origin so that

$$
Z_{\text {min,max }}^{\varepsilon}\left(0, \gamma^{0}\right)=\left(g_{\min , \max }+O(\varepsilon), L_{1}, \gamma^{0}, \Gamma\right) .
$$

The second order $\mathrm{H}_{2}$ in Lemma 2.1 depends on $\gamma$ and therefore may (and will) break integrability. If we consider this Hamiltonian in Poincaré coordinates (20), it posesses a hyperbolic critical orbit $O\left(L_{2}^{-2}\right)$-close to $(\xi, \eta)=(0,0)$.

To show that $H_{2}$ makes the stable and unstable invariant manifolds of this periodic orbit split, we use the classical Poincaré-Melnikov Theory [Mel63]. More precisely, here we define the Poincaré-Melnikov potential

$$
\begin{aligned}
\mathcal{L}^{\varepsilon}\left(\gamma^{0}\right)= & \int_{0}^{+\infty} H_{2} \circ Z^{\varepsilon}\left(t, \gamma^{0}\right)-H_{2} \circ Z_{\max }^{\varepsilon}\left(t, \gamma^{0}\right) d t \\
& +\int_{-\infty}^{0} H_{2} \circ Z^{\varepsilon}\left(t, \gamma^{0}\right)-H_{2} \circ Z_{\min }^{\varepsilon}\left(t, \gamma^{0}\right) d t,
\end{aligned}
$$

similarly to [DG00]), but taking into account the fact that the first order perturbation does not break integrability.

In other words, we consider as an integrable system $\mathcal{H}_{0}=H_{0}+\varepsilon H_{1}$ and as a perturbation $\mathcal{H}_{1}=H_{2}$ (see Lemma 2.1 and recall that we have taken $\varepsilon=L_{2}^{-1}$ ). In Lemma 3.1 we have obtained the parameterization $Z^{0}$ of the separatrix (see (25)). For the Hamiltonian $\mathcal{H}_{0}$, we have a parameterization of the separatrix $Z^{\varepsilon}\left(t, \gamma^{0}\right)=Z^{0}\left(t, \gamma^{0}\right)+O(\varepsilon)$. The time origin can be chosen so that

$$
Z^{\varepsilon}\left(0, \gamma^{0}\right)=\left(\frac{\pi}{2}, \Gamma \sqrt{\frac{5}{3}}+O(\varepsilon), \gamma^{0}, \Gamma\right),
$$

(recall that by Lemma 5.1, $Z^{0}\left(0, \gamma^{0}\right)=\left(\pi / 2, \Gamma \sqrt{5 / 3}, \gamma^{0}, \Gamma\right)$ ).

In order to determine the splitting of separatrices, we consider the section $g_{1}=\pi / 2$ which is transversal to the flow locally in the neigborhood ot the unperturbed separatrix. It is still transversal to the perturbed stable and unstable invariant manifolds. We measure the splitting in this section. The distance between of the invariant manifolds is parameterized by $\gamma^{0}$, the value of the $\gamma$ coordinate when $t=0$.

Melnikov Theory ensures that the transversal homoclinic points in the section $g_{1}=$ $\pi / 2$ are $\varepsilon^{2}=L_{2}^{-2}$-close to the non-degenerate critical points of the Poincaré-Melnikov potential. Expanding the potential in powers of $\varepsilon$, we get $\mathcal{L}^{\varepsilon}\left(\gamma^{0}\right)=\mathcal{L}\left(\gamma^{0}\right)+\mathcal{O}(\varepsilon)$ with

$$
\begin{aligned}
\mathcal{L}\left(\gamma^{0}\right)= & \int_{0}^{+\infty} H_{2} \circ Z^{0}\left(t, \gamma^{0}\right)-H_{2} \circ Z_{\max }^{0}\left(t, \gamma^{0}\right) d t \\
& +\int_{-\infty}^{0} H_{2} \circ Z^{0}\left(t, \gamma^{0}\right)-H_{2} Z_{\min }^{0}\left(t, \gamma^{0}\right) d t .
\end{aligned}
$$

Moreover, since $H_{2}$ has a factor $e_{1}$ (see (7)) it vanishes over the periodic orbits $Z_{\min , \max }^{0}$. Thus, using (18) and (25), the potential reads

$$
\begin{aligned}
\mathcal{L}\left(\gamma^{0}\right) & =\int_{-\infty}^{+\infty} H_{2}\left(g_{1}^{h}(t), G_{1}^{h}(t), \gamma^{h}(t), \Gamma\right) d t \\
& =\int_{-\infty}^{+\infty} H_{2}\left(g_{1}^{h}(t), G_{1}^{h}(t), \gamma^{0}+\gamma^{1}(t)+\gamma^{2}(t), \Gamma\right) d t .
\end{aligned}
$$


Melnikov theory then implies that, $\varepsilon$-close to non-degenerate critical points of $\mathcal{L}$, there exist transversal homoclinic points in the section $g_{1}=\pi / 2$. The potential $\mathcal{L}$ naturally depends on parameters $L_{1}$ and $\Gamma$, which vary in the (non-empty) open set

$$
O=\left\{\left(L_{1}, \Gamma\right): L_{1}>0,0<\Gamma<L_{1} \sqrt{3 / 5}\right\} \subset \mathbb{R}^{2} .
$$

Let us write

$$
\mathcal{L}\left(\gamma^{0}\right)=\mathcal{L}_{L_{1}, \Gamma}\left(\gamma^{o}\right)
$$

The next proposition shows that $\mathcal{L}$ necessarily has non-degenerate critical points, except maybe for exceptional values of the parameters.

Proposition 4.1. There exists a non constant real analytic function $\mathcal{L}^{+}: O \rightarrow \mathbb{R}$ such that the potential (31) is of the form

$$
\mathcal{L}_{L_{1}, \Gamma}\left(\gamma^{0}\right)=\mathcal{L}_{L_{1}, \Gamma}^{+} e^{i \gamma_{0}}+\overline{\mathcal{L}}_{L_{1}, \Gamma} e^{-i \gamma_{0}} .
$$

In particular, outside an analytic subset of $O$ of empty interior, $\mathcal{L}^{+}$does not vanish and thus $\mathcal{L}$ (as a function of $\gamma^{0}$ ) has non-degenerate critical points.

This proposition is proven in Section 5 , where the function $\mathcal{L}^{+}$is computed explicitly (see formula (38) ). It is easier to describe the dynamics when the dimension is as low as possible, so let us carry out the symplectic reduction of the flow by time. Since $\dot{\gamma} \neq 0$, define the Poincaré section $\Sigma_{\gamma_{0}}=\left\{\gamma=\gamma_{0}\right\}$ within some fixed energy level, and the corresponding return map, induced by the Hamiltonian (11),

$$
\mathcal{P}_{\gamma_{0}}: \Sigma_{\gamma_{0}} \longrightarrow \Sigma_{\gamma_{0}}
$$

for which the Poincaré coordinates $(\xi, \eta)$ may be used (see (201)). This map has a hyperbolic fixed point $L_{2}^{-2}$-close to the origin with one dimensional stable and unstable invariant manifolds. The classical Melnikov theory applied to the Melnikov potential obtained in Proposition 4.1 entails the following corollary and theorem. Note that the circle $g_{1}=\pi / 2$ locally corresponds to the line $\xi=0$ (see (20) ).

Corollary 4.2. Fix $\left(L_{1}^{0}, \Gamma^{0}\right) \in O$ (defined in (32) ) such that $\mathcal{L}_{L_{1}^{0}, \Gamma^{0}}^{+} \neq 0$ and let $\gamma_{0}^{*}$ be a non-degenerate critical point of $\mathcal{L}_{L_{1}^{0}, \Gamma^{0}}^{+}$. For $L_{2}$ large enough, there exists some $\widetilde{\gamma}_{0}^{*}=\gamma_{0}^{*}+O\left(L_{2}^{-1}\right)$ such that the Poincaré map $\mathcal{P}_{\widetilde{\gamma}_{0}^{*}}$ has a transversal homoclinic point in the line $\{\xi=0\} \subset \Sigma_{\widetilde{\gamma}_{0}^{*}}$.

Recall that all Poincaré maps are conjugate. Therefore, this corollary implies that there are transversal homoclinic points for $\mathcal{P}_{\gamma_{0}}$ for all $\gamma_{0} \in \mathbb{T}$.

The transversality of invariant manifolds given by the corollary a priori refers to the secular Hamiltonian obtained after one step of averaging. Nevertheless, the conclusion holds for the Poincaré maps of the secular Hamiltonian of any finite order. Indeed, higher order averaging only modifies higher orders of the asymptotic expansion of the splitting, while transversality at the first order was entailed by the first order of the expansion, as given by the Poincaré-Melnikov potential. Hence, by considering the analytic set defined by the condition $\mathcal{L}_{L_{1}^{0}, \Gamma^{0}}^{+} \neq 0$ at all orders of averaging, one gets the main result, where we restrict to some fixed compact set

$$
K \subset O=\left\{\left(L_{1}, \Gamma\right): L_{1}>0,0<\Gamma<L_{1} \sqrt{3 / 5}\right\} \subset \mathbb{R}^{2} .
$$

Theorem 4.3. Fix any $\gamma_{0} \in \mathbb{T}$. There is an analytic set $K^{o}$ of full measure in $K$ such that, for parameters $\left(L_{1}^{0}, \Gamma^{0}\right)$ in $K^{o}$, for $L_{2}$ large enough, the Poincaré maps $\mathcal{P}_{\gamma_{0}}: \Sigma_{\gamma_{0}} \mapsto$ $\Sigma_{\gamma_{0}}$ of the secular systems of all orders posess a horseshoe. 


\subsection{Splitting of separatrices for the non-averaged Hamiltonian}

As explained in Remark 3.2, for the secular Hamiltonian we have a hyperbolic periodic orbit at each energy level within a compact interval of such levels. These periodic orbits form a normally hyperbolic invariant cylinder. Corollary 4.2 implies that the invariant manifolds of this cylinder intersect transversally.

In Section 5 we consider $L_{1}$ and $L_{2}$ as constants. One can lift the dynamics to the extended phase space, with the additional Keplerian coordinates $\left(\ell_{1}, L_{1}, \ell_{2}, L_{2}\right)$. Then, the cylinder gets enlarged by four extra dimensions: $\left(L_{1}, L_{2}\right)$, which are just constants of motion, and $\left(\ell_{1}, \ell_{2}\right)$, which are performing a rigid rotation.

This extended system, after rescaling, is just the first order of (3), that is

$$
F_{0}=F_{\text {Kep }}+F_{\text {sec }} .
$$

Call $\Lambda$ the cylinder of this Hamiltonian. Now one would like to analyze Hamiltonian (3), that is, the full (non averaged) three-body problem. The first step is to prove the persistence of the invariant cylinder when $L_{2}$ is large enough, using that the reminder is $O\left(L_{2}^{-k}\right)$ for some $k \in \mathbb{N}$. Since we are in a singular perturbation regime, the classical theory of persistence of normally hyperbolic invariant manifolds [Fen72 cannot be applied directly. However, now there are results which deal with singularly perturbed problems and which can be applied to the present setting Yan09, GdILT15. Note that we can do global averaging up to high order. Therefore, one does not need to face the problems in [BKZ11] and the obtained cylinder can be as smooth as needed. Call $\widetilde{\Lambda}$ the perturbed cylinder.

In this setting, one can expect Arnold diffusion i.e., orbits whose action components drift by a large amount, uniformly with respect to large $L_{2}$ 's. Since

- $G_{1}$ is prescribed by $\widetilde{\Lambda}$ and the homoclinic channel,

- $G_{2}$ (resp. $L_{1}$ ) is constrained by the conservation of the angular momentum (resp. the energy),

one would expect to obtain orbits with drift in $L_{2}$, that is in the semi major axis of the outer body. This is a remarkable feature, since semi major axes are known to be very stable when two of the three masses are very small [Nie96].

To obtain unstable orbits one usually combines two types of dynamics. The "inner dynamics" is the dynamics of Hamiltonian (3) in restriction to the cylinder $\widetilde{\Lambda}$. The "outer dynamics" and is the so-called scattering map DdlLS08, obtained as the following limit. Assume that the invariant manifolds of $\widetilde{\Lambda}$ split transversally along a homoclinic chanel. Consider a homoclinic orbit in the chanel, which is asymptotic to the trajectory of some point $x_{-} \in \widetilde{\Lambda}$ as $t \rightarrow-\infty$ and to the trajectory of some other point $x_{+} \in \widetilde{\Lambda}$ as $t \rightarrow+\infty$. Then, we say that the scattering map $\mathcal{S}$ maps $x_{-}$to $x_{+}$. hat $\mathcal{S}$ be a map (as opposed to a more general correspondance) is proved in DdILS08] under general hypotheses which are satisfied here. Note that this map depends on the chosen homoclinic chanel and therefore it may not be defined globally -usually it is multivaluated. Provided that these two maps do not have common invariant circles, by iterating them in a random order, one gets pseudo-orbits which have the wanted unstable behavior. A shadowing argument then permits to approximately realize these pseudo-orbits as real orbits of the system.

Understanding both the inner and outer dynamics is certainly not easy for Hamiltonian (3). Concerning the inner dynamics, Jefferys and Moser [JM66] have used KAM theory to show that this cylinder contains quasiperiodic hyperbolic tori which form a 
positive measure set. There should be a very rich dynamics in the complement of these tori in the cylinder. In particular, since the Hamiltonian restricted to the cylinder has three degrees freedom, there may exist Arnold diffusion in the cylinder itself.

Concerning the outer dynamics, one needs first to prove that the invariant manifolds of the cylinder $\widetilde{\Lambda}$ split transversally and then derive some precise dynamical behavior for the scattering map, as in DdlLS08. The analysis in the present paper allows us to perform only the first step.

Theorem 4.4. Fix $L_{1}^{+}>L_{1}^{-}>0$. Consider the Hamiltonian (3) with $L_{1} \in\left[L_{1}^{-}, L_{1}^{+}\right]$ and $L_{2} \geq L_{2}^{0}$. If $L_{2}^{0}$ is large enough,

1. there exists a normally hyperbolic invariant cylinder $\widetilde{\Lambda}$,

2. the invariant manifolds of the cylinder $\widetilde{\Lambda}$ intersect transversally along a homoclinic chanel, which is diffeomorphic to $\widetilde{\Lambda}$,

3. and there exists a scattering map $\mathcal{S}: \widetilde{\Lambda} \longrightarrow \widetilde{\Lambda}$ associated to this homoclinic chanel.

Note that, in the above statement, the cylinder is invariant in the sense that the vector field is tangent to the cylinder, but orbits may escape from its boundary (sometimes one rather refers to these manifolds as weakly invariant).

Proof. As explained above, the persistence of the invariant cylinder can be obtained by the available results of persistence of normally hyperbolic invariant cylinders in the singular perturbation setting [Yan09, GdlLT15].

For the other statements, we may use the results in Proposition 4.1 and DdlLS08. Using the expression of $F_{\mathrm{sec}}$ in (5), one can split the Hamiltonian (3) as $F=H_{0}+H_{1}$ with $H_{0}=F_{\text {Kep }}-\mu_{1} m_{2} F_{\text {quad }}$ and $H_{1}=F-H_{0}$. Therefore,

$$
\begin{aligned}
H_{1}= & -\mu_{1} m_{2}\left(\sigma_{0}-\sigma_{1}\right) F_{\text {oct }}+O\left(\frac{a_{1}^{4}}{a_{2}^{5}}\right) \\
& -\mu_{1} m_{2}\left(\sigma_{0}-\sigma_{1}\right) F_{\text {oct }}+O\left(L_{2}^{-5}\right) .
\end{aligned}
$$

and therefore satisfies $H_{1}=O\left(L_{2}^{-2}\right)$.

Let us abuse notation and assume that $H_{0}$ and $H_{1}$ are expressed in the variables given in (9) and that time has been suitably scaled. As we have explained the Hamiltonian $H_{0}$ has as normally hyperbolic invariant cylinder. Recall that $L_{1}, L_{2}$ and $\Gamma$ are first integrals of $H_{0}$. Thus, the dynamics in this cylinder is foliated by three dimensional invariant tori. Let us define $Y_{0} \equiv Y_{0}\left(t, \ell_{1}^{0}, L_{1}^{0}, \ell_{2}^{0}, L_{2}^{0}, \gamma^{0}, \Gamma^{0}\right)$ the trajectory on the invariant tori $L=L_{i}^{0}$, and $\Gamma=\Gamma^{0}$ in the cylinder with initial condition in the $\left(\ell_{1}, \ell_{2}, \gamma\right)$ variables given by $\left(\ell_{1}^{0}, \ell_{2}^{0}, \gamma^{0}\right)$.

Since $H_{0}$ is integrable, the stable and unstable invariant manifolds of the cylinder agree, and form a homoclinic manifold. The latter is given (at the first order) by the homoclinic of the quadrupolar Hamiltonian obtained in Lemma 3.1 in the space $\left(g_{1}, G_{1}, \gamma, \Gamma\right)$. Recall that for $H_{0}$ the variables $L_{1}$ and $L_{2}$ are first integrals. The dynamics of the variables $\ell_{1}$ and $\ell_{2}$ is close to a rigid rotation and can be easily deduced. These homoclinic manifold can be parameterized by time $t$ and the coordinates of the cylinder $Y \equiv Y\left(t, \ell_{1}^{0}, L_{1}^{0}, \ell_{2}^{0}, L_{2}^{0}, \gamma^{0}, \Gamma^{0}\right)$. Note that $Y$ can be asymptotic to different points in the cylinder as $t \longrightarrow \pm \infty$. There exist smooth functions $\left(\ell_{1}^{ \pm}, \ell_{2}^{ \pm}, \gamma^{ \pm}\right)=\left(\ell_{1}^{ \pm}, \ell_{2}^{ \pm}, \gamma^{ \pm}\right)\left(\ell_{1}^{0}, L_{1}^{0}, \ell_{2}^{0}, L_{2}^{0}, \gamma^{0}, \Gamma^{0}\right)$ such that

$$
Y\left(t, \ell_{1}^{0}, L_{1}^{0}, \ell_{2}^{0}, L_{2}^{0}, \gamma^{0}, \Gamma^{0}\right)-Y_{0}\left(t, \ell_{1}^{ \pm}, L_{1}^{0}, \ell_{2}^{ \pm}, L_{2}^{0}, \gamma^{ \pm}, \Gamma^{0}\right) \longrightarrow 0
$$


as $t \longrightarrow \pm \infty$.

Then, to prove that the invariant manifolds split and that one can define the scattering map of the full Hamiltonian $H_{0}+H_{1}$, one may apply the results in [DdlLS08] (see also [DdlLS06]). Define the Poincaré-Melnikov potential

$$
\widetilde{\mathcal{L}}\left(\ell_{1}^{0}, L_{1}^{0}, \ell_{2}^{0}, L_{2}^{0}, \gamma^{0}, \Gamma^{0}\right)=\int_{-\infty}^{0} H_{1} \circ Y-H_{1} \circ Y_{0}^{-} d t+\int_{0}^{+\infty} H_{1} \circ Y-H_{1} \circ Y_{0}^{+} d t,
$$

where $Y$ stands for $Y\left(t, \ell_{1}^{0}, L_{1}^{0}, \ell_{2}^{0}, L_{2}^{0}, \gamma^{0}, \Gamma^{0}\right)$ and $Y_{0}^{ \pm}$for $Y_{0}\left(t, \ell_{1}^{ \pm}, L_{1}^{0}, \ell_{2}^{ \pm}, L_{2}^{0}, \gamma^{ \pm}, \Gamma^{0}\right)$.

Consider the function

$$
\tau \mapsto \widetilde{\mathcal{L}}\left(\ell_{1}^{0}+\omega_{1} \tau, L_{1}^{0}, \ell_{2}^{0}+\omega_{2} \tau, L_{2}^{0}, \gamma^{0}+\omega_{3} \tau, \Gamma^{0}\right)
$$

where $\omega=\left(\omega_{1}, \omega_{2}, \omega_{3}\right)$ is the frequency vector associated with the torus $L=L_{i}^{0}$, and $\Gamma=\Gamma^{0}$. Results in [DdlLS08] imply that each non-degenerate critical point of this function gives rise to a transversal intersection of the invariant manifolds. The nondegeneracy of the critical point allows us to define a local scattering map.

Using the formula of $H_{1}$, we see that

$$
\widetilde{\mathcal{L}}=\mathcal{L}+O\left(L_{2}^{-2}\right) .
$$

where $\mathcal{L}$ is the Melnikov potential introduced in (31). Proposition 4.1 implies that, as long as $\mathcal{L}_{L_{1}^{0}, \Gamma^{0}}^{+} \neq 0$, the function $\mathcal{L}\left(\gamma^{0}-\omega_{1} \tau\right)$ has non-degenerate critical points with respect to $\tau$. Thanks to the non-degeneracy, the function (33) has critical points, which are $O\left(L_{2}^{-2}\right)$-close to those of $\mathcal{L}\left(\gamma^{0}-\omega_{1} \tau\right)$. Each critical point of (33) gives rise to a transversal intersections and to an associated scattering map.

Theorem 4.4 implies that Hamiltonian (3) fits in the classical framework of Arnold diffusion along single resonances: a normally hyperbolic invariant cylinder whose invariant manifolds intersect transversally [DdlLS00, DdlLS06, BKZ11]. Nevertheless, the results obtained in this paper are not enough to obtain unstable orbits drifting along the cylinder. Indeed, we cannot derive formulas for the scattering map and therefore we have no information about the outer dynamics beyond the fact that it is well defined.

The reason is the following. To prove that the invariant manifolds of the cylinder split, it is enough to project them into a certain plane and see that they intersect transversally in this plane. But, in order to build unstable orbits, one needs more precise information on the scattering map. Namely, to derive a first order approximation of the scattering map, one needs to analyze how the invariant manifolds of the cylinder split in every direction (see [DdlLS08]), in particular in the $L_{1}$ and $L_{2}$ directions. This would give the size of the possible jumps that the scattering maps makes in these directions. That is, how far can be two points in the cylinder which are connected by a heteroclinic orbit. Since the mean anomalies $\ell_{1}$ and $\ell_{2}$ are rapidly oscillating in our regime, the transversality in the conjugate directions $L_{1}$ and $L_{2}$ is exponentially small and therefore very difficult to detect.

An intermediate step would be to tackle the 1-averaged Hamiltonian, where only the fastest mean anomaly $\ell_{1}$ has been averaged out. Then, one has a three degree of freedom Hamiltonian system with only one fast frequency. As a starter, we provide the expression of the analogue to the quadrupolar term:

$$
-\frac{\mu_{1} m_{2}}{\left\|q_{2}\right\|} \int_{\mathbb{T}} \sigma_{2} P_{2}(\cos \zeta) \frac{\left\|q_{1}\right\|^{2}}{\left\|q_{2}\right\|^{3}} d \ell_{1}=
$$




$$
-\frac{3 \mu_{1} m_{2}}{4 a_{1}^{2} r_{2}^{3}}\left(\begin{array}{l}
\left(\left(4 e_{1}^{2}+1\right) \sin ^{2} g_{1}+\left(1-e_{1}^{2}\right) \cos ^{2} g_{1}\right) \cos ^{2} i \sin ^{2}\left(v_{2}+g_{2}\right)+ \\
10 e_{1}^{2} \cos g_{1} \sin g_{1} \cos i \cos \left(v_{2}+g_{2}\right) \sin \left(v_{2}+g_{2}\right)+ \\
\left(\left(1-e_{1}^{2}\right) \sin ^{2} g_{1}+\left(4 e_{1}^{2}+1\right) \cos ^{2} g_{1}\right) \cos ^{2}\left(v_{2}+g_{2}\right) \\
-e_{1}^{2}-\frac{2}{3}
\end{array}\right) .
$$

where $v_{2} \equiv v_{2}\left(G_{2}, L_{2}, \ell_{2}\right)$ is the eccentric anomaly.

Analyzing how the invariant manifolds split either for the full three-body problem or for the 1-averaged Hamiltonian, and deriving formulas for the corresponding scattering maps, would be the major step towards proving Arnold diffusion in the three body problem in the lunar regime.

\section{Proof of Proposition 4.1}

In Lemma 3.1, we have called $\left(g_{1}^{h}, G_{1}^{h}, \gamma^{h}=\gamma^{0}+\gamma^{1}+\gamma^{2}, \Gamma^{h} \equiv \Gamma\right)$ the separatrix solution 4 We compute the potential defined in (31). To this end, we expand it in Fourier series in $\gamma^{0}$. Since $\mathrm{H}_{2}$ is a trigonometric polynomial of degree 1 in $\gamma$ (or equivalently in $g_{2}$, see equation (7)), it can be written as

$$
H_{2}\left(g_{1}, G_{1}, \gamma, \Gamma\right)=H_{2}^{+}\left(g_{1}, G_{1}, \Gamma\right) e^{i \gamma}+H_{2}^{-}\left(g_{1}, G_{1}, \Gamma\right) e^{-i \gamma} .
$$

Because $\gamma^{h}=\gamma^{0}+\gamma^{1}(t)+\gamma^{2}(t)$ depends linearly on $\gamma^{0}, \mathcal{L}\left(\gamma^{0}\right)$ too has only two harmonics:

$$
\mathcal{L}\left(\gamma_{0}\right)=\mathcal{L}^{+} e^{i \gamma^{0}}+\mathcal{L}^{-} e^{-i \gamma^{0}}
$$

where

$$
\mathcal{L}^{ \pm}=\int_{-\infty}^{\infty} H_{2}^{ \pm}\left(g_{1}^{h}(t), G_{1}^{h}(t), \Gamma\right) e^{ \pm i\left(\gamma^{1}(t)+\gamma^{2}(t)\right)} d t .
$$

Since $\mathcal{L}$ is a real function, it is determined by, say, the positive harmonic $\mathcal{L}^{+}=\overline{\mathcal{L}}^{-}$.

As a first step, we parameterize the separatrix by $g_{1}$ instead of $t$, using the following lemma (where we omit the upper index $h$ ).

Lemma 5.1. The following identites are satisfied on the separatrix given by Lemma 3.1;

$$
\begin{cases}G_{1} & =|\Gamma| \sqrt{\frac{5}{3}} \frac{\sin g_{1}}{\sqrt{1-\frac{5}{3} \cos ^{2} g_{1}}} \\ e_{1} & =\sqrt{\frac{2}{3}} \frac{\Gamma}{L_{1} \chi} \sqrt{\frac{1-\frac{5}{3}\left(1+\chi^{2}\right) \cos ^{2} g_{1}}{1-\frac{5}{3} \cos ^{2} g_{1}}} \\ \cos i & =\sqrt{\frac{3}{5}} \frac{\sqrt{1-\frac{5}{3} \cos ^{2} g_{1}}}{\sin g_{1}} \\ \sin i & =\sqrt{\frac{2}{5}} \frac{1}{\sin g_{1}} \\ \cos \gamma^{2} & =\sqrt{1-\frac{5}{3} \cos ^{2} g_{1}} \\ \sin \gamma^{2} & =-\sqrt{\frac{5}{3}} \cos g_{1} .\end{cases}
$$

The proof of this lemma is a direct consequence of Lemma 3.1 and formulas (8), (10), (13). We can use this lemma to express the function $\mathcal{F}^{+}=H_{2}^{+} e^{i \gamma_{2}}$ on the separatrix as a function of $g_{1}$.

\footnotetext{
${ }^{4}$ The variable $t$ used in section 3 is a rescaled time, since in Section 3 we have simplified the quadrupolar Hamiltonian by some multiplicative constant, when replacing $F_{\text {quad }}$ by $H_{0}$.
} 
Lemma 5.2. The function $\mathcal{F}^{+}$can be written, on the separatrix, as a function of $g_{1}$ as $\mathcal{F}^{+}=\frac{1}{2}\left(\mathcal{F}_{1}+i \mathcal{F}_{2}\right)$ with

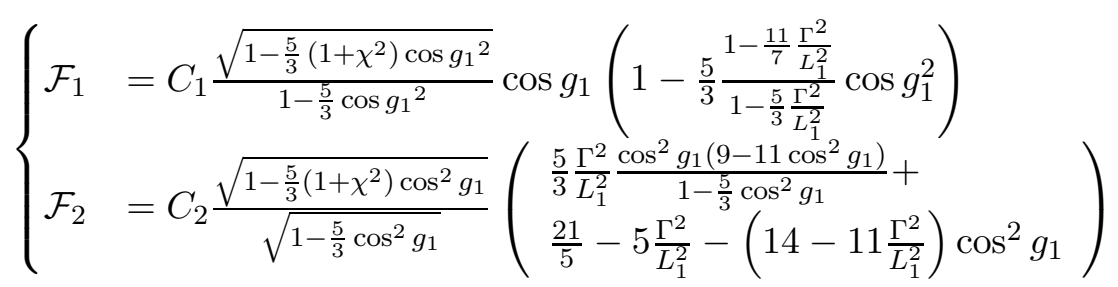

and

$C_{1}=\frac{105}{32} \frac{a_{1}^{3}}{a_{2}^{4}} \frac{e_{2}}{\left(1-e_{2}^{2}\right)^{5 / 2}}\left(1-\frac{5}{3} \frac{\Gamma^{2}}{L_{1}^{2}}\right)^{3 / 2} \quad$ and $\quad C_{2}=\frac{15}{64} \sqrt{\frac{5}{3}} \frac{a_{1}^{3}}{a_{2}^{4}} \frac{e_{2}}{\left(1-e_{2}^{2}\right)^{5 / 2}}\left(1-\frac{5}{3} \frac{\Gamma^{2}}{L_{1}^{2}}\right)^{1 / 2}$.

Proof. We have

$$
H_{2}=A_{\mathrm{oct}} e_{1}\left(\cos g_{1} \cos \gamma A+\sin g_{1} \sin \gamma \cos i B\right)
$$

where

$$
\left\{\begin{array}{l}
A=\frac{G_{1}^{2}}{L_{1}^{2}}\left(5 \sin ^{2} i\left(-7 \cos ^{2} g_{1}+6\right)-3\right)-35 \sin ^{2} g_{1} \sin ^{2} i+7 \\
B=\frac{G_{1}^{2}}{L_{1}^{2}}\left(5 \sin ^{2} i\left(7 \cos ^{2} g_{1}-4\right)+3\right)+35 \sin ^{2} g_{1} \sin ^{2} i-7
\end{array}\right.
$$

and

$$
A_{\mathrm{oct}}=-\frac{15}{64} \frac{a_{1}^{3}}{a_{2}^{4}} \frac{e_{2}}{\left(1-e_{2}^{2}\right)^{5 / 2}}
$$

Thus,

$$
\begin{aligned}
\mathcal{F}^{+}=\frac{A_{\mathrm{oct}} e_{1}}{2}[ & \left(A \cos g_{1} \cos \gamma_{2}+B \sin g_{1} \sin \gamma_{2} \cos i\right) \\
& \left.+i\left(A \cos g_{1} \sin \gamma_{2}-B \sin g_{1} \cos \gamma_{2} \cos i\right)\right],
\end{aligned}
$$

which we want to express in terms of $g_{1}$. Using Lemma 5.1, the functions $A$ and $B$ can be written as

$$
\left\{\begin{array}{l}
A=\frac{5}{3} \frac{\Gamma^{2}}{L_{1}^{2}} \frac{1}{1-\frac{5}{3} \cos ^{2} g_{1}}\left(9-11 \cos ^{2} g_{1}\right)-7 \\
B=-\frac{5}{3} \frac{\Gamma^{2}}{L_{1}^{2}} \frac{1}{1-\frac{5}{3} \cos ^{2} g_{1}}\left(5-11 \cos ^{2} g_{1}\right)+7
\end{array}\right.
$$

Let

$$
\left\{\begin{array}{l}
S_{1}=e_{1}\left(\cos g_{1} \cos \gamma_{2} A+\sin g_{1} \sin \gamma_{2} \cos i B\right) \\
S_{2}=e_{1}\left(\cos g_{1} \sin \gamma_{2} A-\sin g_{1} \cos \gamma_{2} \cos i B\right) .
\end{array}\right.
$$

Then,

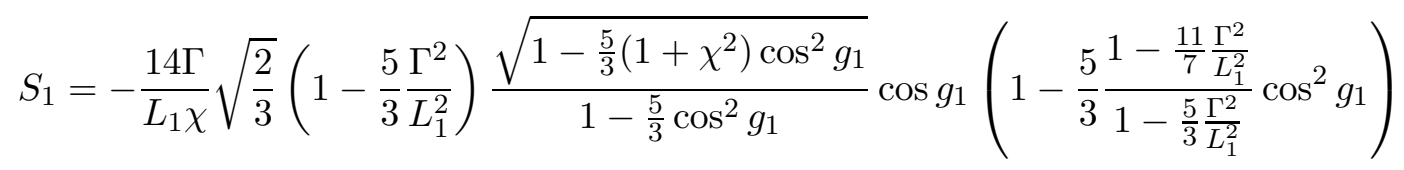

and

$$
\begin{aligned}
S_{2}= & -\frac{\sqrt{10}}{3} \frac{\Gamma}{L_{1} \chi} \frac{\sqrt{1-\frac{5}{3}\left(1+\chi^{2}\right) \cos ^{2} g_{1}}}{\sqrt{1-\frac{5}{3} \cos ^{2} g_{1}}} \times \\
& \left(\frac{5}{3} \frac{\Gamma^{2}}{L_{1}^{2}} \frac{\cos ^{2} g_{1}\left(9-11 \cos ^{2} g_{1}\right)}{1-\frac{5}{3} \cos ^{2} g_{1}}+\frac{21}{5}-5 \frac{\Gamma^{2}}{L_{1}^{2}}-\left(14-11 \frac{\Gamma^{2}}{L_{1}^{2}}\right) \cos ^{2} g_{1}\right) .
\end{aligned}
$$


Now we compute $\mathcal{F}^{+}$as a function of $t$. Recall that the constant $A_{2}$ was defined in (23).

Lemma 5.3. The functions $\mathcal{F}_{1}$ and $\mathcal{F}_{2}$ on the heteroclinic (cf. Lemma 5.2), can be written as functions of $\tau=A_{2} t$ as

$$
\left\{\begin{aligned}
\mathcal{F}_{1}= & \widetilde{C}_{1} \frac{\sinh \tau}{1+\sinh \tau} \cdot \frac{7+6 \sinh ^{2} \tau}{\chi^{2}+\left(1+\chi^{2}\right) \sinh ^{2} \tau} \\
\mathcal{F}_{2}= & C_{2} \frac{1}{\cosh \tau}\left[\frac{21}{5}-5 \frac{\Gamma^{2}}{L_{1}^{2}}+\right. \\
& \left.\frac{\sinh ^{2} \tau}{\chi^{2}+\left(1+\chi^{2}\right) \sinh ^{2} \tau}\left(\frac{\Gamma^{2}}{L_{1}^{2} \chi^{2}} \frac{9 \chi^{2}+\left(\frac{12}{5}+9 \chi^{2}\right) \sinh ^{2} \tau}{\cosh ^{2} \tau}-\frac{3}{5}\left(14-11 \frac{\Gamma^{2}}{L_{1}^{2}}\right)\right)\right]
\end{aligned}\right.
$$

with

$$
\widetilde{C}_{1}=-\frac{15}{16 \sqrt{10}} \frac{a_{1}^{3}}{a_{2}^{4}} \frac{e_{2}}{\left(1-e_{2}^{2}\right)^{5 / 2}} \frac{\Gamma}{L_{1}}\left(1-\frac{5}{3} \frac{\Gamma^{2}}{L_{1}^{2}}\right) .
$$

Proof. We compute each term as a function of $\tau$. By (26), we have that

$$
\sqrt{1-\frac{5}{3}\left(1+\chi^{2}\right) \cos g_{1}^{0}(t)}=\frac{\chi}{\sqrt{\chi^{2}+\left(1+\chi^{2}\right) \sinh ^{2} \tau}}
$$

and

$$
1-\frac{5}{3} \cos g_{1}^{0}(t)=\chi^{2} \frac{1+\sinh ^{2} \tau}{\chi^{2}+\left(1+\chi^{2}\right) \sinh ^{2} \tau} .
$$

So,

$$
\frac{\sqrt{1-\frac{5}{3}\left(1+\chi^{2}\right) \cos g_{1}^{0}(t)} \cos g_{1}^{0}(t)}{1-\frac{5}{3} \cos g_{1}^{0}(t)}=\frac{1}{\chi} \sqrt{\frac{3}{5}} \frac{\sinh \tau}{1+\sinh ^{2} \tau} .
$$

For the last term, we have

$$
\left(1-\frac{5}{3} \frac{1-\frac{11}{7} \frac{\Gamma^{2}}{L_{1}^{2}}}{1-\frac{5}{3} \frac{\Gamma^{2}}{L_{1}^{2}}} \cos ^{2} g_{1}^{0}(t)\right)=\frac{\left(1-\frac{5}{3} \frac{\Gamma^{2}}{L_{1}^{2}}\right) \chi^{2}+M \sinh ^{2} \tau}{\left(1-\frac{5}{3} \frac{\Gamma^{2}}{L_{1}^{2}}\right)\left(\chi^{2}+\left(1+\chi^{2}\right) \sinh ^{2} \tau\right)}
$$

where

$$
M=\left(1-\frac{5}{3} \frac{\Gamma^{2}}{L_{1}^{2}}\right)\left(1+\chi^{2}\right)-\left(1-\frac{11}{7} \frac{\Gamma^{2}}{L_{1}^{2}}\right) .
$$

Using the definition of $\chi$ in (23), we have that

$$
\begin{aligned}
\left(1-\frac{5}{3} \frac{\Gamma^{2}}{L_{1}^{2}}\right) \chi^{2} & =\frac{2}{3} \frac{\Gamma^{2}}{L_{1}^{2}} \\
M & =\frac{4}{7} \frac{\Gamma^{2}}{L_{1}^{2}} .
\end{aligned}
$$

Therefore,

$$
\left(1-\frac{5}{3} \frac{1-\frac{11}{7} \frac{\Gamma^{2}}{L_{1}^{2}}}{1-\frac{5}{3} \frac{\Gamma^{2}}{L_{1}^{2}}} \cos ^{2} g_{1}^{0}(t)\right)=\frac{2 \Gamma^{2}}{21 L_{1}^{2}\left(1-\frac{5}{3} \frac{\Gamma^{2}}{L_{1}^{2}}\right)} \frac{7+6 \sinh ^{2} \tau}{\chi^{2}+\left(1+\chi^{2}\right) \sinh ^{2} \tau} .
$$

Putting all these formulas together and defining

$$
\widetilde{C}_{1}=\frac{2}{21 \chi} C_{1} \sqrt{\frac{3}{5}} \frac{\Gamma^{2}}{L_{1}^{2}} \frac{1}{1-\frac{5}{3} \frac{\Gamma^{2}}{L_{1}^{2}}}
$$

we complete the proof. 
The formulas of Lemma 5.3 allow us to compute the Poincaré - Melnikov potential (34). We split it as as $\mathcal{L}^{+}=\mathcal{L}_{1}+i \mathcal{L}_{2}$ with

$$
\mathcal{L}_{j}=\frac{1}{2} \int_{\mathbb{R}} \mathcal{F}_{j}(t) e^{i \gamma^{1}(t)} d t, j=1,2
$$

We first compute

$$
\begin{aligned}
\mathcal{L}_{1} & =\widetilde{C}_{1} \int_{-\infty}^{\infty} \frac{\sinh \left(A_{2} t\right)}{1+\sinh ^{2}\left(A_{2} t\right)} \cdot \frac{7+6 \sinh ^{2}\left(A_{2} t\right)}{\chi^{2}+\left(1+\chi^{2}\right) \sinh ^{2}\left(A_{2} t\right)} e^{-i \frac{2 \Gamma}{L_{1}^{2}} t} d t \\
& =\frac{\widetilde{C}_{1}}{A_{2}} \int_{-\infty}^{\infty} \frac{\sinh \tau}{1+\sinh ^{2} \tau} \cdot \frac{7+6 \sinh ^{2} \tau}{\chi^{2}+\left(1+\chi^{2}\right) \sinh ^{2} \tau} e^{-i \frac{2 \Gamma}{A_{2} L_{1}^{2}} \tau} d \tau .
\end{aligned}
$$

using the residue theorem. First note that if we look at this integral along the path $\tau=-i \pi+\sigma, \sigma \in \mathbb{R}$, instead of the real line, we get

$$
\begin{aligned}
\int_{-i \pi+\mathbb{R}} & \frac{\sinh \tau}{1+\sinh ^{2} \tau} \cdot \frac{7+6 \sinh ^{2} \tau}{\chi^{2}+\left(1+\chi^{2}\right) \sinh ^{2} \tau} e^{-i \frac{2 \Gamma}{A_{2} L_{1}^{2}} \tau} d \tau \\
=-e^{-\frac{2 \Gamma \pi}{A_{2} L_{1}^{2}}} & \int_{\mathbb{R}} \frac{\sinh \sigma}{1+\sinh ^{2} \sigma} \cdot \frac{7+6 \sinh ^{2} \sigma}{\chi^{2}+\left(1+\chi^{2}\right) \sinh ^{2} \sigma} e^{-i \frac{2 \Gamma}{A_{2} L_{1}^{2}} \sigma} d \sigma .
\end{aligned}
$$

So, if we define the function

$$
f_{1}(\tau)=\frac{\sinh \tau}{1+\sinh ^{2} \tau} \cdot \frac{7+6 \sinh ^{2} \tau}{\chi^{2}+\left(1+\chi^{2}\right) \sinh ^{2} \tau} e^{-i \frac{2 \Gamma}{A_{2} L_{1}^{2}} \tau}
$$

Cauchy's integral formula shows

$$
\mathcal{L}_{1}=-\frac{2 \pi i}{1+e^{-\frac{2 \Gamma \pi}{A_{2} L_{1}^{2}}}} \sum \text { Residues }
$$

where the summation stands over the residues of the function $f_{1}$ in $-\pi<\operatorname{Im} \tau<0$; the negative sign comes from the index of the curve.

Lemma 5.4. The function $f_{1}$ defined in (36) has three singularities in the region $-\pi<$ $\operatorname{Im} \tau<0$ given by

$$
\left\{\begin{array}{l}
a_{0}^{-}=-i \frac{\pi}{2} \\
a_{1}^{-}=-i \arcsin \frac{\chi}{\sqrt{1+\chi^{2}}} \\
a_{2}^{-}=-i\left(\pi+\arcsin \frac{\chi}{\sqrt{1+\chi^{2}}}\right)
\end{array}\right.
$$

and the associated residues are

$$
\left\{\begin{array}{l}
\operatorname{Res}\left(f, a_{0}^{-}\right)=-\frac{2 \Gamma}{A_{2}^{2}} e^{-\frac{\pi \Gamma}{A_{2} L_{1}^{2}}} \\
\operatorname{Res}\left(f, a_{1}^{-}\right)=\frac{7+\chi^{2}}{2 \sqrt{1+\chi^{2}}} e^{-\frac{2 \Gamma}{A_{2} L_{1}^{2}} \arcsin \frac{\chi}{\sqrt{1+\chi^{2}}}} \\
\operatorname{Res}\left(f, a_{2}^{-}\right)=-\frac{7+\chi^{2}}{2 \sqrt{1+\chi^{2}}} e^{-\frac{2 \Gamma}{A_{2} L_{1}^{2}}\left(\pi-\arcsin \frac{\chi}{\sqrt{1+\chi^{2}}}\right)} .
\end{array}\right.
$$

Before proving the lemma, we proceed to deduce the value of the real part of the Melnikov integral. The lemma shows that the sum of formula (37) is

$$
\sum \text { Residues }=-\frac{2 \Gamma}{A_{2} L_{1}^{2}} e^{-\frac{\pi \Gamma}{A_{2} L_{1}^{2}}}+\frac{7+\chi^{2}}{2 \sqrt{1+\chi^{2}}} e^{-\frac{2 \Gamma}{A_{2} L_{1}^{2}} \arcsin \frac{\chi}{\sqrt{1+\chi^{2}}}}\left(1-e^{-\frac{2 \Gamma}{A_{2} L_{1}^{2}} \pi}\right),
$$


hence the function $\mathcal{L}_{1}$ introduced in (35) satisfies

$$
\mathcal{L}_{1}=-\frac{2 \pi i}{1+e^{-\frac{2 \Gamma \pi}{A_{2} L_{1}^{2}}}} \frac{\widetilde{C}_{1}}{A_{2}}\left(\begin{array}{l}
-\frac{2 \Gamma}{A_{2} L_{1}^{2}} e^{-\frac{\pi \Gamma}{A_{2} L_{1}^{2}}}+ \\
+\frac{7+\chi^{2}}{2 \sqrt{1+\chi^{2}}} e^{-\frac{2 \Gamma}{A_{2} L_{1}^{2}} \arcsin \frac{\chi}{\sqrt{1+\chi^{2}}}}\left(1-e^{-\frac{2 \Gamma}{A_{2} L_{1}^{2}} \pi}\right)
\end{array}\right) .
$$

Proof of Lemma 5.4. The singularities are the solutions of

$$
1+\sinh ^{2} \tau=0 \text { or either } \chi^{2}+\left(1+\chi^{2}\right) \sinh ^{2} \tau=0 .
$$

The first equation is just $1+\sinh ^{2} \tau=\cosh ^{2} \tau=0$ and the only possible solution in $-\pi<\operatorname{Im} \tau<0$ is $a_{0}^{-}=-i \pi / 2$. For the second equation, one can take $\tau=i \sigma$. Then, it is equivalent to

$$
\sin \sigma= \pm \frac{\chi}{\sqrt{1+\chi^{2}}}
$$

which gives the other two singularities.

Now we compute the residues. We start by $a_{0}^{-}$. We compute the Laurent series of each term in $f$. From the fact that

$$
\sinh \tau=-i\left(1+\mathcal{O}_{2}(\tau+i \pi / 2)\right)
$$

one can easily deduce the following,

$$
\begin{aligned}
\frac{1}{\cosh ^{2} \tau} & =-\frac{1}{(\tau+i \pi / 2)^{2}}\left(1+\mathcal{O}_{2}(\tau+i \pi / 2)\right) \\
7+6 \sinh ^{2} \tau & =1+\mathcal{O}_{2}(\tau+i \pi / 2) \\
\chi^{2}+\left(1+\chi^{2}\right) \sinh ^{2} \tau & =-1+\mathcal{O}_{2}(\tau+i \pi / 2) .
\end{aligned}
$$

Therefore

$$
\frac{\sinh \tau}{1+\sinh ^{2} \tau} \cdot \frac{7+6 \sinh ^{2} \tau}{\chi^{2}+\left(1+\chi^{2}\right) \sinh ^{2} \tau}=-\frac{i}{(\tau+i \pi / 2)^{2}}\left(1+\mathcal{O}_{2}(\tau+i \pi / 2)\right) .
$$

For the exponential, we know have that

$$
e^{-i \frac{2 \Gamma}{A_{2} L_{1}^{2}} \tau}=e^{-\frac{\pi \Gamma}{A_{2} L_{1}^{2}}}-i \frac{2 \Gamma}{A_{2} L_{1}^{2}} e^{-\frac{\pi \Gamma}{A_{2} L_{1}^{2}}}(\tau+i \pi / 2)+\mathcal{O}_{2}(\tau+i \pi / 2) .
$$

From these two last expansions, we obtain the residue for $a_{0}^{-}=-i \pi / 2$.

Now we compute the other two residues. We compute them at the same time. Note that for $i=1,2$,

$$
\sinh a_{i}^{-}=-i \frac{\chi}{\sqrt{1+\chi^{2}}}
$$

and

$$
\cosh a_{1}^{-}=\frac{1}{\sqrt{1+\chi^{2}}}, \quad \cosh a_{2}^{-}=-\frac{1}{\sqrt{1+\chi^{2}}} .
$$

Therefore

$$
\begin{aligned}
7+6 \sinh ^{2} \tau & =\frac{7+\chi^{2}}{1+\chi^{2}}+\mathcal{O}_{1}(\tau-i \pi / 2) \\
\chi^{2}+\left(1+\chi^{2}\right) \sinh ^{2} \tau & =-2 \chi i\left(\tau-a_{1}^{-}\right)+\mathcal{O}_{2}\left(\tau-a_{1}^{-}\right) \\
\chi^{2}+\left(1+\chi^{2}\right) \sinh ^{2} \tau & =+2 \chi i\left(\tau-a_{2}^{-}\right)+\mathcal{O}_{2}\left(\tau-a_{2}^{-}\right)
\end{aligned}
$$


and we have also

$$
\begin{aligned}
& e^{-i \frac{2 \Gamma}{A_{2} L_{1}^{2}} \tau}=e^{-\frac{2 \Gamma}{A_{2} L_{1}^{2}} \arcsin \frac{\chi}{\sqrt{1+\chi^{2}}}+\mathcal{O}_{1}\left(\tau-a_{1}^{-}\right)} \\
& e^{-i \frac{2 \Gamma}{A_{2} L_{1}^{2}} \tau}=e^{-\frac{2 \Gamma}{A_{2} L_{1}^{2}}\left(\pi-\arcsin \frac{\chi}{\sqrt{1+\chi^{2}}}\right)}+\mathcal{O}_{1}\left(\tau-a_{2}^{-}\right) .
\end{aligned}
$$

With all these expansions, one can easily deduce the last two residues.

Analogously, the function $\mathcal{L}_{2}$ introduced in (35), satisfies

$$
\mathcal{L}_{2}=-\frac{C_{2}}{A_{2}} \frac{2 \pi i}{1+e^{-\frac{2 \Gamma \pi}{A_{2} L_{1}^{2}}}} \sum \text { Residues }
$$

where the sum stands over all residues in $-\pi<\tau<0$ of

$$
\begin{aligned}
f_{2}(\tau)= & \frac{e^{-i \frac{2 \Gamma}{A_{2} L_{1}^{2}} \tau}}{\cosh \tau}\left[\frac{21}{5}-5 \frac{\Gamma^{2}}{L_{1}^{2}}+\right. \\
& \left.\frac{\sinh ^{2} \tau}{\chi^{2}+\left(1+\chi^{2}\right) \sinh ^{2} \tau}\left(\frac{\Gamma^{2}}{L_{1}^{2} \chi^{2}} \frac{9 \chi^{2}+\left(\frac{12}{5}+9 \chi^{2}\right) \sinh ^{2} \tau}{\cosh ^{2} \tau}-\frac{3}{5}\left(14-11 \frac{\Gamma^{2}}{L_{1}^{2}}\right)\right)\right] .
\end{aligned}
$$

Lemma 5.5. In the region $-\pi<\operatorname{Im} \tau<0$, the function $f_{2}$ has the same singularities as $f_{1}$, given in Lemma 5.4. Moreover, the associated residues are given by

$$
\left\{\begin{array}{l}
\operatorname{Res}\left(f_{2}, a_{0}^{-}\right)=-\frac{i}{5}\left(21-8 \frac{\Gamma^{2}}{L_{1}^{2}}+24 \frac{\Gamma^{4}}{A_{2}^{2} L_{1}^{6} \chi^{2}}\right) e^{-\frac{\pi \Gamma}{A_{2} L_{1}^{2}}} \\
\operatorname{Res}\left(f_{2}, a_{1}^{-}\right)=-i \frac{3}{5} \frac{\chi}{\left(1+\chi^{2}\right)^{3 / 2}}\left(11 \frac{\Gamma^{2}}{L_{1}^{2}}-7\right) e^{-\frac{2 \Gamma}{A_{2}^{2}} \arcsin \frac{\chi}{\sqrt{1+\chi^{2}}}} \\
\operatorname{Res}\left(f_{2}, a_{2}^{-}\right)=-i \frac{3}{5} \frac{\chi}{\left(1+\chi^{2}\right)^{3 / 2}}\left(11 \frac{\Gamma^{2}}{L_{1}^{2}}-7\right) e^{-\frac{2 \Gamma}{A_{2}^{2}}\left(\pi-\arcsin \frac{\chi}{\sqrt{1+\chi^{2}}}\right)}
\end{array}\right.
$$

Hence, the function $\mathcal{L}_{2}$ introduced in (35) satisfies

$$
\mathcal{L}_{2}=-\frac{C_{2}}{A_{2}} \frac{2 \pi i}{1+e^{-\frac{2 \Gamma \pi}{A_{2} L_{1}^{2}}}}\left(\begin{array}{l}
-\frac{i}{5}\left(21-8 \frac{\Gamma^{2}}{L_{1}^{2}}+24 \frac{\Gamma^{4}}{A_{2}^{2} L_{1}^{6} \chi^{2}}\right) e^{-\frac{\pi \Gamma}{A_{2}^{2}}}+ \\
-i \frac{3}{5} \frac{\chi}{\left(1+\chi^{2}\right)^{3 / 2}}\left(11 \frac{\Gamma^{2}}{L_{1}^{2}}-7\right) e^{-\frac{2 \Gamma}{A_{2}^{2}} \arcsin \frac{\chi}{\sqrt{1+\chi^{2}}}+} \\
-i \frac{3}{5} \frac{\chi}{\left(1+\chi^{2}\right)^{3 / 2}}\left(11 \frac{\Gamma^{2}}{L_{1}^{2}}-7\right) e^{-\frac{2 \Gamma}{A_{2}^{2}}\left(\pi-\arcsin \frac{\chi}{\sqrt{1+\chi^{2}}}\right)}
\end{array}\right) .
$$

Proof of Lemma 5.5. We use the expansions obtained in the proof of Lemma 5.4. For the last two residues, it is enough to use also that

$$
\frac{\Gamma^{2}}{L_{1}^{2} \chi^{2}} \frac{9 \chi^{2}+\left(\frac{12}{5}+9 \chi^{2}\right) \sinh ^{2} \tau}{\cosh ^{2} \tau}=\frac{33}{5} \frac{\Gamma^{2}}{L_{1}^{2}}+\mathcal{O}\left(\tau-a_{i}^{-}\right)
$$

for $i=1,2$. Then, using the expansions of Lemma 5.4, we can deduce the two last residues.

Now we compute the residue at $\tau=a_{0}$. We split $f_{2}$ into three parts $f_{2}=h_{1}+h_{2}+h_{3}$ with

$$
\left\{\begin{array}{l}
h_{1}(\tau)=\left(\frac{21}{5}-5 \frac{\Gamma^{2}}{L_{1}^{2}}\right) \frac{e^{-i \frac{2 \Gamma}{A_{2} L_{1}^{2}} \tau}}{\cosh \tau} \\
h_{2}(\tau)=-\frac{3}{5}\left(14-11 \frac{\Gamma^{2}}{L_{1}^{2}}\right) \frac{\sinh ^{2} \tau}{\chi^{2}+\left(1+\chi^{2}\right) \sinh ^{2} \tau} \frac{e^{-i \frac{2 \Gamma}{A_{2} L_{1}^{2}}} \frac{\cosh \tau}{-i \frac{2 \Gamma}{A_{2} L_{1}^{2}} \tau}}{\cosh _{3}^{3} \tau}
\end{array}\right.
$$


Functions $h_{1}$ and $h_{2}$ have a pole of order one at $\tau=a_{0}^{-}$so one can proceed as for the others singularies. However, $h_{3}$ has a pole of order 3 and therefore, we need to compute the expansion up to order 3 of each term in $h_{2}$.

We use the expansions

$$
\left\{\begin{array}{l}
\sinh \tau=-i-\frac{i}{2} y^{2}+\mathcal{O}_{4}(y) \\
\cosh \tau=-i y-\frac{i}{6} y^{3}+\mathcal{O}_{5}(y)
\end{array}\right.
$$

where $y=\tau+i \frac{\pi}{2}$. We use this notation throughout the proof.

We start with $h_{1}$ and $h_{2}$. It can be easily seen that

$$
\left\{\begin{array}{l}
\operatorname{Res}\left(h_{1}, a_{0}^{-}\right)=i\left(\frac{21}{5}-5 \frac{\Gamma^{2}}{L_{1}^{2}}\right) e^{-\frac{\pi \Gamma}{A_{2} L_{1}^{2}}} \\
\operatorname{Res}\left(h_{2}, a_{0}^{-}\right)=-i \frac{3}{5}\left(14-11 \frac{\Gamma^{2}}{L_{1}^{2}}\right) e^{-\frac{\pi \Gamma}{A_{2} L_{1}^{2}}}
\end{array}\right.
$$

For $h_{3}$ we use the following expasions

$$
\begin{aligned}
\frac{9 \chi^{2}+\left(\frac{12}{5}+9 \chi^{2}\right) \sinh ^{2} \tau}{\chi^{2}+\left(1+\chi^{2}\right) \sinh ^{2} \tau} \sinh ^{2} \tau & =-\frac{12}{5}-\left(\frac{12}{5}+\frac{33}{5} \chi^{2}\right) y^{2}+\mathcal{O}_{4}(y) \\
\frac{1}{\cosh ^{3} \tau} & =-\frac{i}{y^{3}}\left(1-\frac{y^{2}}{2}+\mathcal{O}_{4}(y)\right) \\
e^{-i \frac{2 \Gamma}{A_{2} L_{1}^{2}} \tau} & =e^{-\frac{\pi \Gamma}{A_{2} L_{1}^{2}}}\left(1-i \frac{2 \Gamma}{A_{2} L_{1}^{2}} y-\frac{2 \Gamma^{2}}{A_{2}^{2} L_{1}^{4}} y^{2}+\mathcal{O}_{4}(y)\right) .
\end{aligned}
$$

Putting together all these expansions one can deduce the residue

$$
\operatorname{Res}\left(h_{3}, a_{0}^{-}\right)=i \frac{\Gamma^{2}}{L_{1}^{2} \chi^{2}}\left(\frac{33}{5} \chi^{2}-\frac{24}{5} \frac{\Gamma^{2}}{A_{2}^{2} L_{1}^{4}}\right) e^{-\frac{\pi \Gamma}{A_{2} L_{1}^{2}}}
$$

Now it only remains to add the three residues to obtain the residue of $f_{2}$ at $\tau=a_{0}^{-}$.

Gathering what precedes, and letting

$$
\alpha=\frac{\pi \Gamma}{A_{2} L_{1}^{2}}, \quad \hat{\Gamma}=\frac{\Gamma}{L_{1}}, \quad \hat{\chi}=\frac{\chi}{\sqrt{1+\chi^{2}}} \quad \text { and } \quad \beta=\frac{\alpha}{\pi} \arcsin \hat{\chi},
$$

we obtain the following analytic expression:

$$
\mathcal{L}^{+}=-\frac{2 \pi i}{A_{2}\left(1+e^{-2 \alpha}\right)}\left(\begin{array}{c}
\widetilde{C}_{1}\left(\begin{array}{l}
-\frac{2 \alpha}{\pi} e^{-\alpha}+ \\
+\frac{7+\chi^{2}}{2 \chi} \sin \frac{\beta \pi}{\alpha} e^{-2 \beta}\left(1-e^{-2 \alpha}\right)
\end{array}\right) \\
+C_{2}\left(\begin{array}{c}
\frac{1}{5}\left(21-8 \hat{\Gamma}^{2}+24 \hat{\Gamma}^{2} \alpha^{2}\right) e^{-\alpha}+ \\
+\frac{3}{5} \frac{\chi}{\left(1+\chi^{2}\right)^{3 / 2}}\left(11 \hat{\Gamma}^{2}-7\right) e^{-2 \beta}+ \\
+\frac{3}{5} \frac{\chi}{\left(1+\chi^{2}\right)^{3 / 2}}\left(11 \hat{\Gamma}^{2}-7\right) e^{-2(\alpha-\beta)}
\end{array}\right)
\end{array}\right) .
$$

In order to check that $\mathcal{L}^{+}$is not consant, notice that, asymptotically when $\Gamma$ tends to 0 (and $L_{1}$ is kept constant), $\alpha=O\left(\Gamma^{-2}\right)$ while $\beta=O\left(\Gamma^{-1}\right)$, so that the term in $\tilde{C}_{1} e^{-2 \beta}$ dominates the others: for some $C_{3}\left(L_{1}\right)$ and $C_{4}\left(L_{1}\right)$ independant of $\Gamma$,

$$
\mathcal{L}^{+} \sim \frac{C_{3}\left(L_{1}\right)}{\Gamma^{2}} \exp \left(-2 C_{4}\left(L_{1}\right) \Gamma+O\left(\Gamma^{-2}\right)\right) .
$$


Since the dominant term of the right hand side is a non constant function of $\Gamma$, Proposition 4.1 is proved.

Funding: J. F. is partially supported by the French ANR (projet ANR-10-BLAN 0102 DynPDE). M. G. is partially supported by the Spanish MINECO-FEDER Grant MTM2012-31714 and the Catalan Grant 2014SGR504.

Conflict of Interest: The authors declare that they have no conflict of interest.

\section{References}

[Ale69] V. M. Alekseev. Quasirandom dynamical systems. Mat. Zametki, 6:489498, 1969.

[BKZ11] P. Bernard, V. Kaloshin, and K. Zhang. Arnold diffusion in arbitrary degrees of freedom and crumpled 3-dimensional normally hyperbolic invariant cylinders. Preprint available at http://arxiv.org/abs/1112.2773, 2011.

[BL10] G. Boué and J. Laskar. A collisionless scenario for Uranus tilting. Astrophysics J. Letters, 712(1):L44, 2010.

[BLF12] G. Boué, J. Laskar, and F. Farago. A simple model of the chaotic eccentricity of mercury. Astron. ES Astrophysics, 2012.

[Bol06] S. Bolotin. Symbolic dynamics of almost collision orbits and skew products of symplectic maps. Nonlinearity, 19(9):2041-2063, 2006.

[DdlLS00] A. Delshams, R. de la Llave, and T.M. Seara. A geometric approach to the existence of orbits with unbounded energy in generic periodic perturbations by a potential of generic geodesic flows of $\mathbb{T}^{2}$. Comm. Math. Phys., 209(2):353-392, 2000.

[DdlLS06] A. Delshams, R. de la Llave, and T.M. Seara. A geometric mechanism for diffusion in hamiltonian systems overcoming the large gap problem: heuristics and rigorous verification on a model. Mem. Amer. Math. Soc., 2006.

[DdlLS08] A. Delshams, R. de la Llave, and T. M. Seara. Geometric properties of the scattering map of a normally hyperbolic invariant manifold. Adv. Math., 217(3):1096-1153, 2008.

[DG00] A. Delshams and P. Gutiérrez. Splitting potential and the PoincaréMelnikov method for whiskered tori in Hamiltonian systems. J. Nonlinear Sci., 10(4):433-476, 2000.

[DKdlRS14] A. Delshams, V. Kaloshin, A. de la Rosa, and T. Seara. Parabolic orbits in the restricted three body problem. Preprint, available at http://arxiv.org/abs/1501.01214, 2014.

[Féj02] J. Féjoz. Quasiperiodic motions in the planar three-body problem. $J$. Differential Equations, 183(2):303-341, 2002.

[Fen72] N. Fenichel. Persistence and smoothness of invariant manifolds for flows. Indiana Univ. Math. J., 21:193-226, 1971/1972. 
[FGKR15] J. Féjoz, M. Guardià, V. Kaloshin, and P. Roldán. Kirkwood gaps and diffusion along mean motion resonances

in the restricted planar three-body problem. to appear, J. Eur. Math. Soc., 2015 .

[FS89] C. Froeschle and H. Scholl. The three principal secular resonances nu(5), $\mathrm{nu}(6)$, and $\mathrm{nu}(16)$ in the asteroidal belt. Celestial Mechanics and Dynamical Astronomy, 46:231-251, September 1989.

[GdlLT15] M. Guardia, R. de la Llave, and R. Treviño. Normally hyperbolic invariant manifolds in hilbert spaces. Work in progress, 2015.

[GMS13] M. Guardia, P. Martín, and T. M. Seara. Homoclinic solutions to infinity and oscillatory motions in the restricted planar circular three body problem. In Progress and challenges in dynamical systems, volume 54 of Springer Proc. Math. Stat., pages 265-280. Springer, Heidelberg, 2013.

[JM66] W. H. Jefferys and J. Moser. Quasi-periodic solutions for the three-body problem. Astronom. J., 71:568-578, 1966.

[Las08] J. Laskar. Chaotic diffusion in the Solar System. Icarus, 196(1):1-15, 2008.

[LR93] J. Laskar and Ph. Robutel. The chaotic obliquity of the planets. Nature, 361(6413):608-612, 1993.

[LS80a] J. Llibre and C. Simó. Oscillatory solutions in the planar restricted threebody problem. Math. Ann., 248(2):153-184, 1980.

[LS80b] J. Llibre and C. Simó. Some homoclinic phenomena in the three-body problem. J. Differential Equations, 37(3):444-465, 1980.

[Mel63] V. K. Melnikov. On the stability of the center for time periodic perturbations. Trans. Moscow Math. Soc., 12:1-57, 1963.

[Moe89] R. Moeckel. Chaotic dynamics near triple collision. Arch. Rational Mech. Anal., 107(1):37-69, 1989.

[Mos01] J. Moser. Stable and random motions in dynamical systems. Princeton Landmarks in Mathematics. Princeton University Press, Princeton, NJ, 2001. With special emphasis on celestial mechanics, Reprint of the 1973 original, With a foreword by Philip J. Holmes.

[Nie96] L. Niederman. Stability over exponentially long times in the planetary problem. Nonlinearity, 9(6):1703-1751, 1996.

[Sit60] K. Sitnikov. The existence of oscillatory motions in the three-body problems. Soviet Physics. Dokl., 5:647-650, 1960.

[Yan09] D. Yang. An invariant manifold for odes and its applications. Preprint available at http://arxiv.org/abs/0909.1103v1, 2009.

[Zha14] L. Zhao. Quasi-periodic almost-collision orbits in the spatial three-body problem. Com. Pure App. Math., 2014. 